\title{
COVERS OF THE INTEGERS WITH ODD MODULI AND THEIR APPLICATIONS TO THE FORMS $x^{m}-2^{n}$ AND $x^{2}-F_{3 n} / 2$
}

\author{
KE-JIAN WU AND ZHI-WEI SUN
}

\begin{abstract}
In this paper we construct a cover $\left\{a_{s}\left(\bmod n_{s}\right)\right\}_{s=1}^{k}$ of $\mathbb{Z}$ with odd moduli such that there are distinct primes $p_{1}, \ldots, p_{k}$ dividing $2^{n_{1}}-1, \ldots, 2^{n_{k}}-$ 1 respectively. Using this cover we show that for any positive integer $m$ divisible by none of $3,5,7,11,13$ there exists an infinite arithmetic progression of positive odd integers the $m$ th powers of whose terms are never of the form $2^{n} \pm p^{a}$ with $a, n \in\{0,1,2, \ldots\}$ and $p$ a prime. We also construct another cover of $\mathbb{Z}$ with odd moduli and use it to prove that $x^{2}-F_{3 n} / 2$ has at least two distinct prime factors whenever $n \in\{0,1,2, \ldots\}$ and $x \equiv a(\bmod M)$, where $\left\{F_{i}\right\}_{i \geqslant 0}$ is the Fibonacci sequence, and $a$ and $M$ are suitable positive integers having 80 decimal digits.
\end{abstract}

\section{INTRODUCTION}

For $a \in \mathbb{Z}$ and $n \in \mathbb{Z}^{+}=\{1,2,3, \ldots\}$ we let

$$
a(n)=\{x \in \mathbb{Z}: x \equiv a(\bmod n)\}
$$

which is a residue class modulo $n$. A finite system

$$
A=\left\{a_{s}\left(n_{s}\right)\right\}_{s=1}^{k}
$$

of residue classes is said to be a cover of $\mathbb{Z}$ if every integer belongs to some members of $A$. Obviously (1.1) covers all the integers if it covers $0,1, \ldots, N_{A}-1$ where $N_{A}=\left[n_{1}, \ldots, n_{k}\right]$ is the least common multiple of the moduli $n_{1}, \ldots, n_{k}$. The reader is referred to $\mathrm{Gu}$, for problems and results on covers of $\mathbb{Z}$ and to [FFKPY] for a recent breakthrough in the field. In this paper we are only interested in applications of covers.

By a known result of Bang $[$ ] (see also Zsigmondy [Z and Birkhoff and Vandiver BV]), for each integer $n>1$ with $n \neq 6$, there exists a prime factor of $2^{n}-1$ not dividing $2^{m}-1$ for any $0<m<n$; such a prime is called a primitive prime divisor of $2^{n}-1$. P. Erdős, who introduced covers of $\mathbb{Z}$ in the early 1930 s, constructed the following cover (cf. E])

$$
A_{0}=\{0(2), 0(3), 1(4), 3(8), 7(12), 23(24)\}
$$

Received by the editor February 15, 2007 and, in revised form, July 4, 2008.

2000 Mathematics Subject Classification. Primary 11B25; Secondary 11A07, 11A41, 11B39, 11D61, $11 \mathrm{Y} 99$.

Key words and phrases. Cover of the integers, arithmetic progression, Fibonacci sequence, prime divisor.

The second author is responsible for communications, and supported by the National Natural Science Foundation (grant 10871087) of People's Republic of China.

(C)2009 American Mathematical Society Reverts to public domain 28 years from publication 
whose moduli are distinct, greater than one and different from 6 . It is easy to check that $2^{2}-1,2^{3}-1,2^{4}-1,2^{8}-1,2^{12}-1,2^{24}-1$ have primitive prime divisors $3,7,5,17,13,241$ respectively. Using the cover $A_{0}$ and the Chinese Remainder Theorem, Erdös showed that any integer $x$ satisfying the congruences

$$
\left\{\begin{array}{l}
x \equiv 2^{0}(\bmod 3) \\
x \equiv 2^{0}(\bmod 7) \\
x \equiv 2^{1}(\bmod 5) \\
x \equiv 2^{3}(\bmod 17) \\
x \equiv 2^{7}(\bmod 13) \\
x \equiv 2^{23}(\bmod 241)
\end{array}\right.
$$

and the additional congruences $x \equiv 1(\bmod 2)$ and $x \equiv 3(\bmod 31)$ cannot be written in the form $2^{n}+p$ with $n \in \mathbb{N}=\{0,1,2, \ldots\}$ and $p$ a prime. The reader may consult [SY] for a refinement of this result. By improving the work of Cohen and Selfridge [CS], Sun [S00] showed that for any integer

$$
x \equiv 47867742232066880047611079\left(\bmod \prod_{p \in P} p\right)
$$

with

$$
P=\{2,3,5,7,11,13,17,19,31,37,41,61,73,97,109,151,241,257,331\},
$$

we have $x \neq \pm p^{a} \pm q^{b}$ where $p, q$ are primes and $a, b \in \mathbb{N}$. In 2005, Luca and Stănică [LS] constructed a cover of $\mathbb{Z}$ to show that if $n$ is sufficiently large and $n \equiv 1807873(\bmod 3543120)$, then $F_{n} \neq p^{a}+q^{b}$ with $p, q$ prime numbers and $a, b \in \mathbb{N}$, where the Fibonacci sequence $\left\{F_{n}\right\}_{n \geqslant 0}$ is given by

$$
F_{0}=0, F_{1}=1 \text {, and } F_{n+1}=F_{n}+F_{n-1} \text { for } n=1,2,3, \ldots \text {. }
$$

A famous conjecture of Erdős and J. L. Selfridge states that there does not exist a cover of $\mathbb{Z}$ with all the moduli odd, distinct and greater than one. There is little progress on this open conjecture (cf. $\mathrm{Gu}$, and $\mathrm{GS}$ ). In contrast, we have the following theorem.

Theorem 1.1. There exists a cover $A_{1}=\left\{a_{s}\left(n_{s}\right)\right\}_{s=1}^{173}$ of $\mathbb{Z}$ with all the moduli greater than one and dividing the odd number

$$
3^{3} \times 5^{2} \times 7 \times 11 \times 13=675675,
$$

for which there are distinct primes $p_{1}, \ldots, p_{173}$ greater than 5 such that each $p_{s}(1 \leqslant$ $s \leqslant 173)$ is a primitive prime divisor of $2^{n_{s}}-1$.

Theorem 1.1 has the following application.

Theorem 1.2. Let $N$ be any positive integer. Then there is a residue class consisting of odd numbers such that for each nonnegative $x$ in the residue class and each $m \in\{1, \ldots, N\}$ divisible by none of $3,5,7,11,13$, the number $x^{m}-2^{n}$ with $n \in \mathbb{N}$ always has at least two distinct prime factors.

Remark 1.1. Let $m \in \mathbb{Z}^{+}$. Chen $[\mathrm{C}]$ conjectured that there are infinitely many positive odd numbers $x$ such that $x^{m}-2^{n}$ with $n \in \mathbb{Z}^{+}$always has at least two distinct prime factors, and he was able to prove this when $m \equiv 1(\bmod 2)$ or $m \equiv \pm 2(\bmod 12)$. The conjecture is particularly difficult when $m$ is a high power of 2. In a recent paper [FFK], Filaseta, Finch and Kozek confirmed the conjecture 
for $m=4,6$ with the help of a deep result of Darmon and Granville [DG] on generalized Fermat equations; they also showed that there exist infinitely many integers $x \in\left\{1,3^{8}, 5^{8}, \ldots\right\}$ such that $x^{m} 2^{n}+1$ with $n \in \mathbb{Z}^{+}$always has at least two distinct prime divisors.

Recall that $\left\{F_{n}\right\}_{n \geqslant 0}$ is the Fibonacci sequence. Set $u_{n}=F_{3 n} / 2$ for $n \in \mathbb{N}$. Clearly, $u_{0}=0, u_{1}=1$, and

$$
\begin{aligned}
u_{n+1} & =\frac{F_{3 n+3}}{2}=\frac{F_{3 n+1}+\left(F_{3 n+1}+F_{3 n}\right)}{2} \\
& =F_{3 n+1}+u_{n}=F_{3 n-1}+3 u_{n} \\
& =4 u_{n}+\frac{2 F_{3 n-1}-F_{3 n}}{2} \\
& =4 u_{n}+\frac{F_{3 n-1}-F_{3 n-2}}{2} \\
& =4 u_{n}+u_{n-1}
\end{aligned}
$$

for every $n=1,2,3, \ldots$.

Now we give the third theorem which is of a new type and will be proved on the basis of a certain cover of $\mathbb{Z}$ with odd moduli.

Theorem 1.3. Let

$$
\begin{aligned}
a= & 312073868852745021881735221320236651673651936708237682^{-} \\
& 34185354856354918873864275
\end{aligned}
$$

and

$$
\begin{aligned}
M= & 368128524439220711844024989130760705031462298208612115^{-} \\
& 58347078871354783744850778 .
\end{aligned}
$$

Then, for any $x \equiv a(\bmod M)$ and $n \in \mathbb{N}$, the number $x^{2}-F_{3 n} / 2$ has at least two distinct prime divisors.

Remark 1.2. (a) Actually our proof of Theorem 1.3 yields the following stronger result: Whenever $y \in a^{2}(M)$ and $n \in \mathbb{N}$, the number $y-F_{3 n} / 2$ has at least two distinct prime divisors.

(b) In view of Theorem 1.3, it is interesting to study the diophantine equation $x^{2}-F_{3 n} / 2= \pm p^{a}$ with $a, n, x \in \mathbb{N}$ and $p$ a prime, or the equation $F_{3 n}=2 x^{2} \pm d y^{2}$ with $d$ equal to 1 or 2 or twice an odd prime. The related equation $F_{n}=x^{2}+d y^{2}$ has been investigated by Ballot and Luca [BL].

The second author has the following conjecture.

Conjecture 1.1. Let $m$ be any positive integer. Then there exist $b, d \in \mathbb{Z}^{+}$such that whenever $x \in b^{m}(d)$ and $n \in \mathbb{N}$ the number $x-F_{n}$ has at least two distinct prime divisors. Also, there are odd integer $b$ and even number $d \in \mathbb{Z}^{+}$such that whenever $x \in b^{m}(d)$ and $n \in \mathbb{N}$ the number $x-2^{n}$ has at least two distinct prime divisors.

Remark 1.3. (a) We are unable to prove Conjecture 1.1 since it is difficult for us to construct a suitable cover of $\mathbb{Z}$ for the purpose.

(b) In 2006, Bugeaud, Mignotte and Siksek BMS showed that the only powers in the Fibonacci sequence are

$$
F_{0}=0, F_{1}=F_{2}=1, F_{6}=2^{3} \text { and } F_{12}=12^{2} .
$$


It seems challenging to solve the diophantine equation $x^{m}-F_{n}= \pm p^{a}$ with $a, n, x \in$ $\mathbb{N}, m>1$, and $p$ a prime.

We are going to show Theorems 1.1-1.3 in Sections 2-4 respectively.

\section{Proving Theorem 1.1 via constructions}

Proof of Theorem 1.1. Let $a_{1}\left(n_{1}\right), \ldots, a_{173}\left(n_{173}\right)$ be the following 173 residue classes respectively.

0(3), 1(5), 0(7), 1(9), 7(11), 8(11), 7(13), 8(15), 19(21), 17(25), 22(25), $25(27), 23(33), 29(35), 30(35), 14(39), 17(39), 4(45), 13(45), 0(55)$, 25(55), 50(55), 25(63), 52(63), 9(65), 2(75), 32(75), 13(77), 41(91), 62(91), 76(91), 5(99), 65(99), 86(99), 44(105), 59(105), 89(105), 31(117), 43(117), 83(117), 103(117), 35(135), 43(135), 88(135), 26(143), 86(143), 125(143), 35(165), 37(175), 87(175), 162(175), 34(189), 53(189), 155(195), 85(225), 130(225), 157(225), 202(225), 137(231), 158(231), 104(273), 146(273), 188(273), 65(275), 175(275), 152(297), 218(297), 79(315), 284(315), 295(315), 87(325), 112(325), 162(325), 16(351), 44(351), 97(351), 286(351), 313(351), 15(385), 225(385), 290(385), 191(429), 203(429), 284(429), 34(455), 454(455), 130(495), 230(495), 395(495), 179(525), 362(525), 445(525), 494(525), 335(585), 355(585), 412(585), 490(585), 7(675), 232(675), 277(675), 502(675), 200(693), 257(693), 515(693), 445(715), 500(715), 555(715), 356(819), 538(819), 629(819), 100(825), 145(825), 265(825), 475(825), 179(945), 494(945), 562(975), 637(975), 662(975), 862(975), 937(975), 115(1001), 808(1001), 5(1155), 809(1155), 845(1155), 950(1155), 614(1287), 742(1287), 1010(1287), 767(1365), 977(1365), 1235(1365), 350(1485), 220(1575), 662(1575), 1012(1575), 1390(1575), 470(1755), 580(1755), 610(1755), 880(1755), 564(1925), 949(1925), 1089(1925), 1334(1925), 1474(1925), 1859(1925), 202(2079), 895(2079), 911(2079), 1105(2145), 1670(2145), 1012(2275), 1362(2275), 1537(2275), 647(2457), 853(2457), 1210(2457), 1214(2457), 2365(2457), 2384(2457), 670(2475), 2245(2475), 2290(2475), 2264(3003), 1390(3465), 416(3861), 3195(5005), 1600(5775), 2920(6435), 7825(10395), 583939(675675).

It is easy to check that the least common multiple of $n_{1}, \ldots, n_{173}$ is the odd number

$$
3^{3} \times 5^{2} \times 7 \times 11 \times 13=675675 .
$$

Since $A_{1}=\left\{a_{s}\left(n_{s}\right)\right\}_{s=1}^{173}$ covers $0, \ldots, 675674$, it covers all the integers.

Using the software Mathematica and the main tables of [BLSTW, pp.1-59], below we associate each $n \in\left\{n_{1}, \ldots, n_{173}\right\}$ with $m_{n}$ distinct primitive prime divisors $p_{n, 1}, \ldots, p_{n, m_{n}}$ of $2^{n}-1$ and write $n: p_{n, 1}, \ldots, p_{n, m_{n}}$ for this, where $m_{n}$ is the 
number of occurrences of $n$ among the moduli $n_{1}, \ldots, n_{173}$. For those

$$
n \in\{1485,3003,3465,3861,5005,5775,6435,10395,675675\},
$$

as $m_{n}=1$ we just need one primitive prime divisor of $2^{n}-1$ whose existence is guaranteed by Bang's theorem; but they are too large to be included in the following list.

$3: 7 ; \quad 5: 31 ; \quad 7: 127 ; \quad 9: 73 ; \quad 11: 23,89 ; \quad 13: 8191 ;$ 15: $151 ; \quad 21: 337 ; \quad 25: 601,1801 ; \quad 27: 262657$; 33: $599479 ; \quad 35: 71,122921 ; \quad 39: 79,121369 ; \quad 45: 631,23311$; 55: 881, 3191, 201961; $\quad 63: 92737,649657$; $\quad 65: 145295143558111$; 75: 100801, 10567201; $\quad$ 77: 581283643249112959; 91: 911, 112901153, 23140471537; 99: 199, 153649, 33057806959; 105: 29191, 106681, 152041; $\quad$ 117: 937, 6553, 86113, 7830118297; 135: 271, 348031, 49971617830801;

143: 724153, 158822951431, 5782172113400990737;

165: 2048568835297380486760231;

175: 39551, 60816001, 535347624791488552837151;

189: 1560007,207617485544258392970753527 ;

195: 134304196845099262572814573351 ;

225: 115201, 617401, 1348206751, 13861369826299351;

231: 463, 4982397651178256151338302204762057;

273: 108749551, 4093204977277417, 86977595801949844993;

275: 382027665134363932751, 4074891477354886815033308087379995347151;

297: 8950393, 170886618823141738081830950807292771648313599433;

315: 870031, 983431, 29728307155963706810228435378401;

325: 7151, 51879585551, 4613679391936953610429590532014122532260339739644049093601;

351: 446473, 29121769, 571890896913727, 93715008807883087, 150832426800173710177 ;

385: 55441, 1971764055031, 31055341681190444478126719755965134571151473925765532041 ;

429: 17286204937, 1065107717756542892882802586807, 167833515549285827885461382441449 ;

455: 200201, 4774797453608343803270988984332214098351782527747577456028391624903856636676854631 ;

495: 991, 334202934764737951438594746151, 6084777159537635796550536863741698483921;

525: 4201, 7351, 181165951, 325985508875527587669607097222667557116221139090131514801;

585: 2400314671, 339175003117573351, 255375215316698521591, 2728334536034592865339299805712535332071;

675: 1605151, 1094270085398478390395590841401, 2842496263188647640089794561760551, 470390038503476855180627941942761032401;

693: 289511839, 2868251407519807, 3225949575089611556532995773813585269068981944367719218489696982054779837928902323497 ;

715: 249602191565465311, 598887853030285391, 40437156024702109576962112690515640573348784018939257192870865875822732631168387328482154414164150624064713711; 
819: 2681001528674743, 219516331727145697249308031, 21494973179303913190133458460563964459380529075838941297352657742148160962406273546512257;

825: 702948566745151, 9115784422509601, 4108316654247271397904922852177568560929751, 101249241260240615605217612230376981800142669401;

945: 124339521078546949914304521499392241, 89371283318924988713544642472309024678004403189516730060412595564942724011446583991926781827601 ;

975: 1951, 8837728285481551, 26155966684789722885001, 1663763381192308635718252801, 4294500770439625509689707482842762056791217143467781867769939979855730352201

1001: 6007, 6952744694636960851412179090394909207;

1155: 2311, 6250631311, 494224324441, 260078892331205274324088366772886790199621606534384599607578416912079166019131912393708208277038936454393545946152508951 ;

1287: 216217, 71477407, 141968533929529744009;

1365: 469561, 52393016292934591, 2224981001722824694441;

1575: 82013401, 32758188751, 76641458269269601, 764384916291005220555242939647951;

1755: 3511, 196911, 4242734772486358591, 85488365519409100951;

1925: 11551, 13167001, 1891705201, 5591298184498951, 292615400703113951, 5627063397043739893603449551 ;

2079: 4159, 16633, 80932047967;

2145: 96001053721, 347878768688881;

2275: 218401, 28319200001, 1970116306308855665077103351;

2457: 565111, 1410319, 21287449, 41194063, 16751168775662428927, 1786131074995391292297656133027144291751;

2475: 4951, 143551, 1086033846151.

Observe that $p_{n, j}>5$ for all $n \in\left\{n_{1}, \ldots, n_{173}\right\}$ and $1 \leqslant j \leqslant m_{n}$. In view of the above, Theorem 1.1 has been proved.

\section{Proof of Theorem 1.2}

Recall that an odd prime $p$ is called a Wieferich prime if $2^{p-1} \equiv 1\left(\bmod p^{2}\right)$. The only known Wieferich primes are 1093 and 3511, and there are no others below $1.25 \times 10^{15}$ (cf. [R, p. 230]).

Suppose that $n \neq 6$ is an integer greater than one, and $p$ is a primitive prime divisor of $2^{n}-1$. Then $n$ is the order of $2 \bmod p$ and hence $p-1$ is a multiple of $n$ by Fermat's little theorem. Thus $2^{n}-1 \mid 2^{p-1}-1$, and hence $p^{2} \nmid 2^{n}-1$ if $p$ is not a Wieferich prime.

Let $A_{1}=\left\{a_{s}\left(n_{s}\right)\right\}_{s=1}^{173}$ and $p_{1}, \ldots, p_{173}$ be as described in Theorem 1.1. For each $s=1, \ldots, 173$ let $q_{s}$ be a primitive prime divisor of $2^{p_{s}^{2}}-1$. Then $p_{1}, \ldots, p_{173}$, $q_{1}, \ldots, q_{173}$ are distinct odd primes since $\left\{p_{1}^{2}, \ldots, p_{173}^{2}\right\} \cap\left\{n_{1}, \ldots, n_{173}\right\}=\emptyset$.

For each $s=1, \ldots, 173$ let $\alpha_{s}$ be the largest positive integer with $p_{s}^{\alpha_{s}} \mid 2^{n_{s}}-1$. Since 3511 is the only Wieferich prime in the set $\left\{p_{1}, \ldots, p_{173}\right\}$, we have $\alpha_{s}=1$ if $p_{s} \neq 3511$. In the case $p_{s}=3511$, we have $\alpha_{s}=2$ since $3511^{2} \mid 2^{3510}-1$, but $3511^{3} \nmid 2^{3510}-1$.

Let $M=2^{2 L} \prod_{s=1}^{173} p_{s}^{\alpha_{s}+2} q_{s}$, where $L$ is the smallest positive integer satisfying

$$
2^{L}-1>\max \left\{16 N, p_{1}^{\alpha_{1}+1}, \ldots, p_{173}^{\alpha_{173}+1}\right\} .
$$


By the Chinese Remainder Theorem, there exists a unique $a \in\{1, \ldots, M\}$ such that

$$
1+3 \cdot 2^{L}\left(2^{2 L}\right) \cap \bigcap_{s=1}^{173}\left(x_{s}^{b_{s}}\left(p_{s}^{\alpha_{s}+2}\right) \cap y_{s}^{b_{s}}\left(q_{s}\right)\right)=a(M) .
$$

Let $m \leqslant N$ be a positive integer relatively prime to $3 \cdot 5 \cdot 7 \cdot 11 \cdot 13=15015$, and write $m=2^{\alpha} m_{0}$ with $\alpha \in \mathbb{N}, m_{0} \in \mathbb{Z}^{+}$and $2 \nmid m_{0}$. Let $s \in\{1, \ldots, 173\}$. Since $n_{s}$ is a divisor of $3^{3} \cdot 5^{2} \cdot 7 \cdot 11 \cdot 13=675675$, we have $\operatorname{gcd}\left(m, n_{s}\right)=1$ and hence $m_{0} b_{s} \equiv a_{s}\left(\bmod n_{s}\right)$ for some $b_{s} \in \mathbb{N}$.

As the order of $2 \bmod p_{s}$ is the odd number $n_{s}, n_{s}$ divides $\left(p_{s}-1\right) / \operatorname{gcd}\left(2^{\alpha}, p_{s}-1\right)$ and hence

$$
2^{\left(p_{s}-1\right) / \operatorname{gcd}\left(2^{\alpha}, p_{s}-1\right)} \equiv 1\left(\bmod p_{s}\right), 2^{p_{s}\left(p_{s}-1\right) / \operatorname{gcd}\left(2^{\alpha}, p_{s}-1\right)} \equiv 1\left(\bmod p_{s}^{2}\right), \ldots
$$

Since there is a primitive root modulo $p_{s}^{\alpha_{s}+2}$ and

$$
2^{\varphi\left(p_{s}^{\alpha_{s}+2}\right) / \operatorname{gcd}\left(2^{\alpha}, \varphi\left(p_{s}^{\alpha_{s}+2}\right)\right)}=2^{p_{s}^{\alpha_{s}+1}\left(p_{s}-1\right) / \operatorname{gcd}\left(2^{\alpha}, p_{s}-1\right)} \equiv 1\left(\bmod p_{s}^{\alpha_{s}+2}\right)
$$

(where $\varphi$ is Euler's totient function), by [IR, Proposition 4.2.1] there exists $x_{s} \in \mathbb{Z}$ with $x_{s}^{2^{\alpha}} \equiv 2\left(\bmod p_{s}^{\alpha_{s}+2}\right)$. Similarly, the order $p_{s}^{2}$ of $2 \bmod q_{s}$ divides $\left(q_{s}-\right.$ $1) / \operatorname{gcd}\left(2^{\alpha}, q_{s}-1\right)$, therefore $2^{\left(q_{s}-1\right) / \operatorname{gcd}\left(2^{\alpha}, q_{s}-1\right)} \equiv 1\left(\bmod q_{s}\right)$ and hence $y_{s}^{2^{\alpha}} \equiv$ $2\left(\bmod q_{s}\right)$ for some $y_{s} \in \mathbb{Z}$.

Let $x \geqslant 0$ be an element of $a(M)$. As $A_{1}$ is a cover of $\mathbb{Z}$, for any $n \in \mathbb{N}$ there is an $s \in\{1, \ldots, 173\}$ such that $n \equiv a_{s}\left(\bmod n_{s}\right)$. Clearly,

$$
x^{m} \equiv\left(x_{s}^{b_{s}}\right)^{m}=\left(x_{s}^{2^{\alpha}}\right)^{m_{0} b_{s}} \equiv 2^{m_{0} b_{s}}\left(\bmod p_{s}^{\alpha_{s}+2}\right),
$$

thus

$$
x^{m}-2^{n} \equiv 2^{m_{0} b_{s}}-2^{a_{s}} \equiv 0\left(\bmod p_{s}^{\alpha_{s}}\right)
$$

since $2^{n_{s}} \equiv 1\left(\bmod p_{s}^{\alpha_{s}}\right)$ and $m_{0} b_{s} \equiv a_{s}\left(\bmod n_{s}\right)$.

As $16 m \leqslant 16 N<2^{L}-1$ and $x \equiv 1+3 \cdot 2^{L}\left(\bmod 2^{2 L}\right)$, we have $\left|x^{m}-2^{n}\right| \geqslant$ $2^{L}-1>p_{s}^{\alpha_{s}+1}$ by [C] Lemma 1]. So $\left|x^{m}-2^{n}\right| \neq 0, p_{s}^{\alpha_{s}}, p_{s}^{\alpha_{s}+1}$. If $x^{m}-2^{n}$ is not divisible by $p_{s}^{\alpha_{s}+2}$, then it must have at least two distinct prime divisors.

Now we assume that $x^{m}-2^{n} \equiv 0\left(\bmod p_{s}^{\alpha_{s}+2}\right)$. Note that $2^{n} \equiv x^{m} \equiv$ $2^{m_{0} b_{s}}\left(\bmod p_{s}^{\alpha_{s}+2}\right)$. Since $n_{s}$ is the order of $2 \bmod p_{s}^{\alpha_{s}}$ and not the order of 2 $\bmod p_{s}^{\alpha_{s}+1}$, by [C, Corollary 3] we have $2^{n} \equiv 2^{m_{0} b_{s}}\left(\bmod q_{s}\right)$. Thus

$$
x^{m}-2^{n} \equiv\left(y_{s}^{b_{s}}\right)^{2^{\alpha} m_{0}}-2^{m_{0} b_{s}} \equiv 0\left(\bmod q_{s}\right)
$$

and so the nonzero integer $x^{m}-2^{n}$ has at least two distinct prime divisors (including $p_{s}$ and $\left.q_{s}\right)$.

By the above, we have proved the desired result.

Remark 3.1. Given $m, n \in \mathbb{Z}^{+}$and an odd prime $p$, the equation $x^{m}-2^{n}=p^{b}$ with $b, x \in \mathbb{N}$ only has finitely many solutions. As observed by the referee, this is a consequence of the Darmon-Granville theorem in [DG. In the case $m=2$, all the finitely many solutions are effectively computable by the algorithms given by Weger [W]. 


\section{Proof of Theorem 1.3}

Lemma 4.1. Let $c \in \mathbb{Z}^{+}$, and define $\left\{U_{n}\right\}_{n \geqslant 0}$ by

$$
U_{0}=0, U_{1}=1 \text {, and } U_{n+1}=c U_{n}+U_{n-1} \text { for } n=1,2,3, \ldots \text {. }
$$

Suppose that $n>0$ is an integer with $n \equiv 2(\bmod 4)$ and $p$ is a prime divisor of $U_{n}$ which divides none of $U_{1}, \ldots, U_{n-1}$. Then $U_{k n+r} \equiv U_{r}(\bmod p)$ for all $k \in \mathbb{N}$ and $r \in\{0, \ldots, n-1\}$.

Proof. By [HS, Lemma 2], $U_{n+1} \equiv-(-1)^{n / 2}=1(\bmod p)$. If $k \in \mathbb{N}$ and $r \in$ $\{0, \ldots, n-1\}$, then $U_{k n+r} \equiv U_{n+1}^{k} U_{r}\left(\bmod U_{n}\right)$ by [HS, Lemma 3] or [S92, Lemma 2], therefore $U_{k n+r} \equiv U_{r}(\bmod p)$.

Proof of Theorem 1.3. Let $b_{1}\left(m_{1}\right), \ldots, b_{24}\left(m_{24}\right)$ be the following 24 residue classes:

$$
\begin{aligned}
& \text { 1(3), 2(5), 3(5), 4(7), 6(7), 0(9), 5(15), 11(15), 9(21), 12(21), } \\
& \text { 1(35), 14(35), 24(35), 29(35),6(45), 15(45), 29(45), 30(45), } \\
& 5(63), 23(63), 44(63), 66(105), 21(315), 89(315) .
\end{aligned}
$$

It is easy to check that $\left\{b_{t}\left(m_{t}\right)\right\}_{t=1}^{24}$ forms a cover of $\mathbb{Z}$ with odd moduli. Set $m_{0}=1$. Then

$$
B=\left\{1\left(2 m_{0}\right), 2 b_{1}\left(2 m_{1}\right), \ldots, 2 b_{24}\left(2 m_{24}\right)\right\}
$$

is a cover of $\mathbb{Z}$ with all the moduli congruent to $2 \bmod 4$.

Let $u_{n}=F_{3 n} / 2$ for $n \in \mathbb{N}$. As we mentioned in Section $1, u_{0}=0, u_{1}=1$ and $u_{n+1}=4 u_{n}+u_{n-1}$ for $n=1,2,3, \ldots$ For a prime $p$ and an integer $n>0$, we call $p$ a primitive prime divisor of $u_{n}$ if $p \mid u_{n}$, but $p \nmid u_{k}$ for those $0<k<n$.

Let $p_{0}, \ldots, p_{24}$ be the following 25 distinct primes respectively:

2, 19, 31, 11, 211, 29, 5779, 541, 181, 31249, 1009, 767131, 21211, 911,

71, 119611, 42391, 271, 811, 379, 912871, 85429, 631, 69931, 17011.

One can easily verify that each $p_{t}(0 \leqslant t \leqslant 24)$ is a primitive prime divisor of $u_{2 m_{t}}$.

The residue class $a(M)$ in Theorem 1.3 is actually the intersection of the following 25 residue classes with the moduli $p_{0}, \ldots, p_{24}$ respectively:

$$
\begin{aligned}
& \text { 1(2), 2(19), 14(31), 4(11), 94(211), 5(29), 0(5779), 156(541), 76(181), } \\
& \text { 10727(31249), 501(1009), 2(767131), 7199(21211), 257(911), 30(71), } \\
& \text { 13909(119611), 9054(42391), 85(271), 292(811), 72(379), 80065(912871), } \\
& \text { 40368(85429), 205(631), 19928(69931), 497(17011). }
\end{aligned}
$$

It is known that the only solutions of the diophantine equation $F_{n}=2 x^{2}$ with $n, x \in \mathbb{N}$ are $(n, x)=(0,0),(3,1),(6,2)$. (Cf. $\quad$ Co, Theorem 4].) Let $x$ be any integer in the residue class $a(M)$. Then $|x|>2$ and hence $x^{2} \neq u_{n}=F_{3 n} / 2$ for all $n \in \mathbb{N}$. With the help of Lemma 4.1 in the case $c=4$, one can check that $x^{2} \equiv u_{1}=1\left(\bmod p_{0}\right)$ and $x^{2} \equiv u_{2 b_{t}}\left(\bmod p_{t}\right)$ for all $t=1, \ldots, 24$.

Let $n$ be any nonnegative integer. As $B$ forms a cover of $\mathbb{Z}, n \equiv 1\left(\bmod 2 m_{0}\right)$ or $n \equiv 2 b_{t}\left(\bmod 2 m_{t}\right)$ for some $1 \leqslant t \leqslant 24$. By Lemma 4.1 with $c=4$, if $n \equiv$ $1\left(\bmod 2 m_{0}\right)$, then $u_{n} \equiv u_{1}=1\left(\bmod p_{0}\right)$ and hence $x^{2}-u_{n} \equiv x^{2}-1 \equiv 0\left(\bmod p_{0}\right)$; if $n \equiv 2 b_{t}\left(\bmod 2 m_{t}\right)$, then $u_{n} \equiv u_{2 b_{t}}\left(\bmod p_{t}\right)$ and hence $x^{2}-u_{n} \equiv x^{2}-u_{2 b_{t}} \equiv$ $0\left(\bmod p_{t}\right)$. Thus, it remains to show that for any given $a, b \in \mathbb{N}$ we can deduce a contradiction if $x^{2}-u_{1+2 m_{0} a}= \pm 2^{b}$ or $x^{2}-u_{2 b_{t}+2 m_{t} a}= \pm p_{t}^{b}$ for some $1 \leqslant t \leqslant 24$. 
Case 4.0. $x^{2}-u_{1+2 a}= \pm 2^{b}$.

As $p_{2}=31$ and $p_{3}=11$ are primitive prime divisors of $u_{2 m_{2}}=u_{2 m_{3}}=u_{10}$, and

$$
u_{1}=1, u_{3}=17, u_{5}=305, u_{7}=5473, u_{9}=98209
$$

have residues $1,-14,-5,-14,1$ modulo 31 and residues $1,-5,-3,-5,1$ modulo 11 respectively. If $2 a+1 \not \equiv 5(\bmod 10)$, then by Lemma 4.1 we have

$$
x^{2}-u_{1+2 a} \equiv 10-1,10-(-14) \not \equiv \pm 1, \pm 2, \pm 4, \pm 8, \pm 16(\bmod 31)
$$

which contradicts $x^{2}-u_{1+2 a}= \pm 2^{b}$. (Note that $2^{5} \equiv 1(\bmod 31)$.) So $2 a+1 \equiv$ $5(\bmod 10)$. It follows that

$x^{2}-u_{1+2 a} \equiv 10-(-5) \equiv-2^{4}(\bmod 31)$ and $x^{2}-u_{1+2 a} \equiv 5-(-3)=2^{3}(\bmod 11)$.

Thus $x^{2}-u_{1+2 a}$ can only be $-2^{b}$ with $b \equiv 4(\bmod 5)$, which cannot be congruent to $2^{3} \bmod 11$. (Note that $2^{5} \equiv-1(\bmod 11)$.) So we have a contradiction.

Case 4.1. $x^{2}-u_{2+6 a}= \pm 19^{b}$.

Observe that

$$
u_{0}=0, u_{2}=4, u_{4}=72, u_{6}=1292, u_{8}=23184
$$

have residues $0,4,-5,5,-4$ modulo 11 and $0,4,10,-10,-4$ modulo 31 respectively. Also, $19^{b} \equiv 2^{3 b} \equiv \pm 1, \pm 2, \pm 4, \pm 3, \pm 5(\bmod 11)$ and $19^{5} \equiv\left(-2^{2} \cdot 3\right)^{5} \equiv-3^{5} \equiv$ $5(\bmod 31)$.

If $2+6 a \equiv 0(\bmod 10)$, then

$$
x^{2}-u_{2+6 a} \equiv 5-0 \equiv 19^{8},-19^{3}(\bmod 11)
$$

and hence $x^{2}-u_{2+6 a}=(-1)^{d-1} 19^{3+5 d}$ for some $d \in \mathbb{N}$, this leads to a contradiction since $x^{2}-u_{2+6 a} \equiv 10-0(\bmod 31)$, but

$$
19^{3+5 d} \equiv 8 \times 5^{d} \equiv 8,9,14 \not \equiv \pm 10(\bmod 31) .
$$

Now we handle the case $2+6 a \equiv 2(\bmod 10)$. Since 181 is a primitive prime divisor of $u_{30}$, and $6 a \equiv 0(\bmod 30)$ and $19^{2} \equiv-1(\bmod 181)$, we have

$$
x^{2}-u_{2+6 a} \equiv 76^{2}-u_{2} \equiv-20 \not \equiv \pm 19^{b}(\bmod 181)
$$

which leads a contradiction.

If $2+6 a \equiv 4(\bmod 10)$, then $x^{2}-u_{2+6 a} \equiv 10-10=0(\bmod 31)$. If $2+6 a \equiv$ $6(\bmod 10)$, then $x^{2}-u_{2+6 a} \equiv 5-5=0(\bmod 11)$. So, when $2+6 a \equiv 4,6(\bmod 10)$ we get a contradiction since $x^{2}-u_{2+6 a}= \pm 19^{b}$.

If $2+6 a \equiv 8(\bmod 10)$, then $x^{2}-u_{2+6 a} \equiv 5-(-4) \equiv 19^{2},-19^{7}(\bmod 11)$ and hence $x^{2}-u_{2+6 a}=(-1)^{d} 19^{2+5 d}$ for some $d \in \mathbb{N}$, this leads to a contradiction since $x^{2}-u_{2+6 a} \equiv 10-(-4) \equiv-11 \times 10(\bmod 31)$, but

$$
19^{2+5 d} \equiv-11 \times 5^{d} \equiv-11,-11 \times 5,-11 \times(-6) \not \equiv \pm 11 \times 10(\bmod 31) .
$$

Case 4.2. $x^{2}-u_{4+10 a}= \pm 31^{b}$.

As $x^{2}-u_{4+10 a} \equiv 5-u_{4} \equiv 5-(-5) \equiv-1(\bmod 11)$ and $31^{b} \equiv(-2)^{b} \equiv$ $1,-2,4,-8,16(\bmod 11)$, we must have $x^{2}-u_{4+10 a}=-31^{b}$ with $b \equiv 0(\bmod 5)$. As $31^{5} \equiv 2^{3}=8(\bmod 19), 31^{b} \equiv 8^{b / 5} \equiv \pm 1, \pm 8, \pm 7(\bmod 19)$. If $3 \nmid a$, then $4+10 a \equiv 0,2(\bmod 6)$ and hence

$$
x^{2}-u_{4+10 a} \equiv 4-u_{0}, 4-u_{2} \equiv 4,0 \not \equiv-31^{b}(\bmod 19) .
$$

Thus $a=3 c$ for some $c \in \mathbb{N}$. As

$$
-8^{b / 5} \equiv-31^{b}=x^{2}-u_{4+10 a} \equiv 4-u_{4}=4-72 \equiv 8(\bmod 19),
$$


we have $b / 5-1 \equiv 3(\bmod 6)$ and hence $b=20+30 d$ for some $d \in \mathbb{N}$. As $31^{10} \equiv-1(\bmod 181)$, we have $31^{b}=31^{20+30 d} \equiv(-1)^{2+3 d}=(-1)^{d}(\bmod 181)$. On the other hand,

$$
-31^{b}=x^{2}-u_{4+10 a}=x^{2}-u_{4+30 c} \equiv 76^{2}-u_{4} \equiv-16-72=-88(\bmod 181) .
$$

So we get a contradiction.

Case 4.3. $x^{2}-u_{6+10 a}= \pm 11^{b}$.

As $x^{2}-u_{6+10 a} \equiv 10-(-10) \equiv-11(\bmod 31)$, and the order of $11 \bmod 31$ is 30 , we have $x^{2}-u_{6+10 a}=(-1)^{d-1} 11^{1+15 d}$ for some $d \in \mathbb{N}$. Since $11^{15} \equiv(-8)^{15}=$ $\left(-2^{9}\right)^{5} \equiv 1(\bmod 19)$, we have $x^{2}-u_{6+10 a} \equiv \pm 11(\bmod 19)$.

If $6+10 a \equiv 0,2(\bmod 6)$, then

$$
x^{2}-u_{6+10 a} \equiv 4-u_{0}, 4-u_{2} \not \equiv \pm 11(\bmod 19) .
$$

So $6+10 a \equiv 4(\bmod 6)$, i.e., $a=1+3 c$ for some $c \in \mathbb{N}$. Therefore,

$$
x^{2}-u_{6+10 a}=x^{2}-u_{16+30 c} \equiv-16-u_{16} \equiv-16-47 \equiv-11 \times 88(\bmod 181) .
$$

Note that

$$
(-11)^{15 d} \equiv(-49)^{d} \equiv 1,-49,48 \not \equiv 88(\bmod 181) .
$$

As $x^{2}-u_{6+10 a}=(-11)^{1+15 d}$, we get a contradiction.

Case 4.4. $x^{2}-u_{8+14 a}= \pm 211^{b}$.

As $p_{5}=29$ is a primitive divisor of $u_{2 m_{5}}=u_{14}$, we have $x^{2}-u_{8+14 a} \equiv 25-u_{8} \equiv$ $25-13=12(\bmod 29)$.

Since 2 is a primitive root $\bmod 29$, also $211 \equiv 2^{3}(\bmod 29), 2^{3 \times 21} \equiv 2^{7} \equiv$ $12(\bmod 29)$, and $2^{3 \times 7} \equiv 12^{3} \equiv-12(\bmod 29)$, we have $x^{2}-u_{8+14 a}=(-1)^{d-1} 211^{7+14 d}$ for some $d \in \mathbb{N}$.

Observe that

$$
\begin{aligned}
x^{2}-u_{8+14 a} & \equiv 10-u_{0}, 10-u_{2}, 10-u_{4}, 10-u_{6}, 10-u_{8}, \\
& \equiv 10-0,10-4,10-10,10-(-10), 10-(-4)(\bmod 31) .
\end{aligned}
$$

Clearly, $211 \equiv 5^{2}(\bmod 31)$ and $5^{3} \equiv 1(\bmod 31)$, thus

$$
211^{7+14 d} \equiv 5^{14+28 d} \equiv 5^{2+d} \equiv-6,1,5(\bmod 31) .
$$

Therefore, $2|d, 3| d$ and $8+14 a \equiv 2(\bmod 10)$. It follows that $a=1+5 c$ for some $c \in \mathbb{N}$ and $d=6 e$ for some $e \in \mathbb{N}$.

Note that

$$
x^{2}-u_{8+14(1+5 c)} \equiv x^{2}-u_{2} \equiv 5-4=1(\bmod 11)
$$

and

$$
(-1)^{d-1} 211^{7+14 d} \equiv-2^{7(1+12 e)} \equiv-2^{7(1+2 e)}(\bmod 11) .
$$

So $2^{7(1+2 e)} \equiv-1 \equiv 2^{5}(\bmod 11)$, hence $7(1+2 e) \equiv 5 \equiv 35(\bmod 10)$ and thus $e \equiv 2(\bmod 5)$. Therefore $7+14 d \equiv 7+84 \times 2 \equiv 35(\bmod 140)$ and hence

$$
211^{7+14 d} \equiv(-2)^{35} \equiv\left(\frac{-2}{71}\right)=-\left(\frac{2}{71}\right)=-1(\bmod 71)
$$

by the theory of quadratic residues, but

$$
x^{2}-u_{8+14 a}=x^{2}-u_{22+70 c} \equiv 30^{2}-u_{22}=900-13888945017644 \equiv 14(\bmod 71),
$$

so we get a contradiction from the equality $x^{2}-u_{8+14 a}=-211^{7+14 d}$. 
Case 4.5. $x^{2}-u_{12+14 a}= \pm 29^{b}$.

As $29^{b} \equiv(-2)^{b} \equiv \pm 1, \pm 2, \pm 4, \pm 8, \pm 16(\bmod 31), x^{2} \equiv 14^{2} \equiv 10(\bmod 31)$ and

$$
u_{12+14 a} \equiv u_{0}, u_{2}, u_{4}, u_{6}, u_{8} \equiv 0,4,10,-10,-4(\bmod 31)
$$

we have $x^{2}-u_{12+14 a} \not \equiv \pm 29^{b}(\bmod 31)$. So, a contradiction occurs.

Case 4.6. $x^{2}-u_{0+18 a}= \pm 5779^{b}$.

As $x^{2}-u_{18 a} \equiv 2^{2}-u_{0}=4(\bmod 19), 5779 \equiv 3(\bmod 19)$ and the order of 3 $\bmod 19$ equals 18 , we have $x^{2}-u_{18 a}=(-1)^{d-1} 5779^{5+9 d}=(-5779)^{5+9 d}$ for some $d \in \mathbb{N}$.

Note that

$$
(-5779)^{9 d} \equiv(-13)^{9 d} \equiv\left(2^{2}\right)^{3 d} \equiv 2^{d} \equiv 1,2,4,8,16(\bmod 31)
$$

and $5779^{5} \equiv 13^{5} \equiv 6(\bmod 31)$. Thus

$$
x^{2}-(-5779)^{5+9 d} \equiv 10+6 \times 2^{d} \equiv-15,-9,3,-4,13(\bmod 31)
$$

while $u_{18 a} \equiv u_{0}, u_{2}, u_{4}, u_{6}, u_{8} \equiv 0,4,10,-10,-4(\bmod 31)$. As $u_{18 a}=x^{2}-$ $(-5779)^{5+9 d}$, we must have $18 a \equiv 8(\bmod 10)$ and $d=3+5 e$ for some $e \in \mathbb{N}$.

Observe that $x^{2}-u_{18 a} \equiv 5-u_{8} \equiv-2(\bmod 11)$, but

$$
(-5779)^{5+9 d} \equiv\left(-2^{2}\right)^{5+9(3+5 e)}=(-1)^{e} 2^{64+90 e} \equiv(-1)^{e} 2^{4} \not \equiv-2(\bmod 11) .
$$

So a contradiction occurs.

Case 4.7. $x^{2}-u_{10+30 a}= \pm 541^{b}$.

As $x^{2}-u_{10+30 a} \equiv 5-u_{0} \equiv 5(\bmod 11)$ and

$$
541^{b} \equiv 2^{b} \equiv \pm 1, \pm 2, \pm 3, \pm 4, \pm 8, \pm 16(\bmod 11),
$$

$x^{2}-u_{10+30 a}=(-1)^{d} 541^{4+5 d}$ for some $d \in \mathbb{N}$, and hence we have a contradiction since $x^{2}-u_{10+30 a} \equiv 10-u_{0}=10(\bmod 31)$, but

$$
541^{4+5 d} \equiv(2 \times 7)^{4+5 d} \equiv 7 \times 5^{d} \equiv 7,7 \times 5,7 \times(-6) \not \equiv \pm 10(\bmod 31) .
$$

Case 4.8. $x^{2}-u_{22+30 a}= \pm 181^{b}$.

As $x^{2}-u_{22+30 a} \equiv 5-u_{2}=5-4(\bmod 11)$ and $181^{b} \equiv 5^{b} \equiv 1,5,3,4,-2(\bmod 11)$, we have $x^{2}-u_{22+30 a}=181^{b}$ with $b=5 d$ for some $d \in \mathbb{N}$. Since $x^{2}-u_{22+30 a} \equiv x^{2}-$ $u_{2} \equiv 10-4=6(\bmod 31)$ and $181^{5 d} \equiv(-5)^{5 d} \equiv 6^{d} \equiv 1,6,5,-1,-6,-5(\bmod 31)$, $d=1+6 e$ for some $e \in \mathbb{N}$. Note that $x^{2}-u_{22+30 a} \equiv 4-u_{4}=4-72 \equiv 8(\bmod 19)$, but

$$
181^{5 d} \equiv(-9)^{5 d}=\left(-3^{10}\right)^{d} \equiv 3^{d}=3^{1+6 e} \equiv 3 \times 7^{e} \equiv 3,2,-5 \not \equiv 8(\bmod 19) .
$$

Case 4.9. $x^{2}-u_{18+42 a}= \pm 31249^{b}$.

Note that $31249^{b} \equiv 1^{b}=1(\bmod 31), x^{2} \equiv 10(\bmod 31)$ and also

$$
u_{18+42 a} \equiv u_{0}, u_{2}, u_{4}, u_{6}, u_{8} \equiv 0,4,10,-10,-4(\bmod 31) .
$$

Therefore, $x^{2}-u_{18+42 a} \not \equiv \pm 31249^{b}(\bmod 31)$.

Case 4.10. $x^{2}-u_{24+42 a}= \pm 1009^{b}$.

As $x^{2}-u_{24+42 a} \equiv 4-u_{0}=4(\bmod 19), 1009 \equiv 2(\bmod 19)$ and 2 is a primitive root modulo 19, we have $x^{2}-u_{24+42 a}=(-1)^{d} 1009^{2+9 d}=(-1009)^{2+9 d}$ for some $d \in \mathbb{N}$. Observe that $u_{10}=416020$ and $x^{2}-u_{24+42 a} \equiv 25-u_{10} \equiv 10(\bmod 29)$, but $6^{7} \equiv-1(\bmod 29)$ and hence

$$
(-1009)^{2+9 d} \equiv 6^{2+9 d} \equiv \pm 1, \pm 6, \pm 7, \pm 13, \pm 9, \pm 4, \pm 5 \not \equiv 10(\bmod 29) .
$$

So we get a contradiction. 
Case 4.11. $x^{2}-u_{2+70 a}= \pm 767131^{b}$.

Observe that $x^{2}-u_{2+70 a} \equiv 5^{2}-u_{2} \equiv-8(\bmod 29)$ and

$$
767131^{b} \equiv(-6)^{b} \equiv 1,-6,7,-13,-9,-4,-5(\bmod 29) .
$$

So a contradiction occurs.

Case 4.12. $x^{2}-u_{28+70 a}= \pm 21211^{b}$.

As $28+70 a \equiv 0(\bmod 14)$, we have $x^{2}-u_{28+70 a} \equiv 5^{2}-u_{0} \equiv-4(\bmod 29)$. On the other hand,

$$
21211^{b} \equiv \pm 12^{b} \equiv \pm 1, \pm 12(\bmod 29) .
$$

Thus, we have a contradiction.

Case 4.13. $x^{2}-u_{48+70 a}= \pm 911^{b}$.

Note that $x^{2}-u_{48+70 a} \equiv 5^{2}-u_{6}=25-1292 \equiv 9(\bmod 29)$, but $911^{b} \equiv 12^{b} \equiv$ $\pm 1, \pm 12(\bmod 29)$.

Case 4.14. $x^{2}-u_{58+70 a}= \pm 71^{b}$.

Observe that $x^{2}-u_{58+70 a} \equiv 5^{2}-u_{2} \equiv-8(\bmod 29)$, but

$$
71^{b} \equiv 13^{b} \equiv \pm 1, \pm 13, \pm 5, \pm 7, \pm 4, \pm 6, \pm 9(\bmod 29) \text {. }
$$

Case 4.15. $x^{2}-u_{12+90 a}= \pm 119611^{b}$.

Since $x^{2}-u_{12+90 a} \equiv 4-u_{0} \equiv 4(\bmod 19)$ and

$$
119611^{b} \equiv 6^{b} \equiv 1,6,-2,7,4,5,-8,9,-3(\bmod 19),
$$

we must have $x^{2}-u_{12+90 a}=119611^{b}$ with $b=4+9 d$ for some $d \in \mathbb{N}$. Note that $x^{2}-u_{12+90 a} \equiv 10-u_{2}=6 \equiv 10 \times 13(\bmod 31)$, but

$$
119611^{4+9 d} \equiv 13^{4+9 d} \equiv 10(-2)^{d}(\bmod 31)
$$

with $(-2)^{d} \equiv \pm 1, \pm 2, \pm 4, \pm 8, \pm 16 \not \equiv 13(\bmod 31)$. So we have a contradiction.

Case 4.16. $x^{2}-u_{30+90 a}= \pm 42391^{b}$.

As $x^{2}-u_{30+90 a} \equiv 4-u_{0}=4(\bmod 19), 42391 \equiv 2(\bmod 19)$ and 2 is a primitive root $\bmod 19$, we have $x^{2}-u_{30+90 a}=(-1)^{d} 42391^{2+9 d}$ for some $d \in \mathbb{N}$.

Note that $x^{2}-u_{30+90 a} \equiv 10-0(\bmod 31)$ and

$$
(-42391)^{2+9 d} \equiv(-14)^{2+9 d} \equiv 10\left(-2^{4}\right)^{3 d} \equiv 10(-1)^{d} 2^{2 d}(\bmod 31) .
$$

Since the only residues of powers of 2 modulo 31 are 1, 2, 4, 8, 16, we must have $x^{2}-u_{30+90 a}=(-42391)^{2+9 d}$ with $d$ divisible by both 5 and 2 . Write $d=10 e$ with $e \in \mathbb{N}$. Then

$$
x^{2}-u_{30+90 a}=42391^{2+90 e} \equiv(-3)^{2+90 e} \equiv 9(\bmod 11),
$$

which contradicts the fact $x^{2}-u_{30+90 a} \equiv 5-u_{0}=5(\bmod 11)$.

Case 4.17. $x^{2}-u_{58+90 a}= \pm 271^{b}$.

Note that $x^{2}-u_{58+90 a} \equiv 10-u_{8} \equiv 14(\bmod 31)$ while

$$
271^{b} \equiv(-2)^{3 b} \equiv \pm 1, \pm 2, \pm 4, \pm 8, \pm 16(\bmod 31) .
$$

Case 4.18. $x^{2}-u_{60+90 a}= \pm 811^{b}$.

As $x^{2}-u_{60+90 a} \equiv 10-u_{0}=10(\bmod 31)$ and $811^{b} \equiv 5^{b} \equiv 1,5,25(\bmod 31)$. we have a contradiction.

Case 4.19. $x^{2}-u_{10+126 a}= \pm 379^{b}$.

Note that $x^{2}-u_{10+126 a} \equiv 2^{2}-u_{4}=4-72 \equiv 8(\bmod 19)$ but $379^{b} \equiv(-1)^{b} \equiv$ $\pm 1(\bmod 19)$. 
Case 4.20. $x^{2}-u_{46+126 a}= \pm 912871^{b}$.

Since $x^{2}-u_{46+126 a} \equiv 2^{2}-u_{4} \equiv 2^{3}(\bmod 19), 912871^{b} \equiv 2^{4 b}(\bmod 19)$ and the order of $2 \bmod 19$ is 18 , we must have $x^{2}-u_{46+126 a}=-912871^{b}$ with $b=3+9 d$ for some $d \in \mathbb{N}$. Note that $x^{2}-u_{46+126 a} \equiv 5^{2}-u_{4}=25-72 \equiv 11(\bmod 29)$, but

$$
912871^{3+9 d} \equiv 3^{2(3+9 d)} \equiv 4^{1+3 d} \equiv \pm 1, \pm 4, \pm 13, \pm 6, \pm 5, \pm 9, \pm 7(\bmod 29) .
$$

So we have a contradiction.

Case 4.21. $x^{2}-u_{88+126 a}= \pm 85429^{b}$.

Observe that $x^{2}-u_{88+126 a} \equiv 5^{2}-u_{4} \equiv 11(\bmod 29)$, but

$$
85429^{b} \equiv(-5)^{b} \equiv 1,-5,-4,-9,-13,7,-6(\bmod 29) .
$$

So a contradiction occurs.

Case 4.22. $x^{2}-u_{132+210 a}= \pm 631^{b}$.

Note that $x^{2}-u_{132+210 a} \equiv 4^{2}-u_{2} \equiv 1(\bmod 11)$ and $631 \equiv 2^{2}(\bmod 11)$. Since $2^{5} \equiv-1(\bmod 11)$ and $2^{10} \equiv 1(\bmod 11)$, we must have $x^{2}-u_{132+210 a}=631^{b}$ with $b=5 d$ for some $d \in \mathbb{N}$. As $x^{2}-u_{132+210 a} \equiv 10-u_{2}=6(\bmod 31), 631^{5} \equiv$ $\left(-2^{2} \times 5\right)^{5} \equiv-5^{2} \equiv 6(\bmod 31)$ and the order of $6 \bmod 31$ is 6 , we can write $d=1+6 e$ with $e \in \mathbb{N}$. Thus

$$
x^{2}-u_{132+210 a}=631^{5+30 e} \equiv\left(2^{2}\right)^{5+30 e} \equiv(-2)^{1+6 e} \equiv-2,5,-3(\bmod 19) .
$$

On the other hand, $x^{2}-u_{132+210 a} \equiv 4-u_{0}=4(\bmod 19)$. This leads to a contradiction.

Case 4.23. $x^{2}-u_{42+630 a}= \pm 69931^{b}$.

As $42+630 a \equiv 0(\bmod 6)$, we have $x^{2}-u_{42+630 a} \equiv 2^{2}-u_{0}=4(\bmod 19)$. On the other hand, $69931^{b} \equiv(-2)^{3 b} \equiv 1,-8,7(\bmod 19)$. So we get a contradiction.

Case 4.24. $x^{2}-u_{178+630 a}= \pm 17011^{b}$.

Since $178+630 a \equiv 10(\bmod 14)$, we have

$$
x^{2}-u_{178+630 a} \equiv 5^{2}-u_{10}=25-416020 \equiv 10(\bmod 29) .
$$

Note that $17011^{b} \equiv(-12)^{b} \equiv \pm 1, \pm 12(\bmod 29)$. So a contradiction occurs.

In view of the above, we have completed the proof of Theorem 1.3.

\section{ACKNOWLEDGMENT}

The authors would like to thank the referee for some helpful comments.

\section{REFERENCES}

[BL] C. Ballot and F. Luca, On the equation $x^{2}+d y^{2}=F_{n}$, Acta Arith. 127 (2007), 145-155. MR2289980 (2008a:11116)

[B] A. S. Bang, Taltheoretiske Undersgelser, Tidsskrift for Mat. 4 (1886), no. 5, 70-80, 130-137.

[BLSTW] J. Brillhart, D. H. Lehmer, J. L. Selfridge, B. Tuckerman, and S. S. Wagstaff, Jr., Factorizations of $b^{n} \pm 1, b=2,3,5,6,7,10,11,12$ up to High Powers, Third ed., Contemporary Mathematics 22, Amer. Math, Soc., Providence, RI, 2002. MR715603 (84k:10005)

[BMS] Y. Bugeaud, M. Mignotte and S. Siksek, Classical and modular approaches to exponential Diophantine equations. I. Fibonacci and Lucas perfect powers, Ann. of Math. (2) 163 (2006), 969-1018. MR2215137 (2007f:11031)

[BV] G. D. Birkhoff and H. S. Vandiver, On the integral divisors of $a^{n}-b^{n}$, Ann. of Math. 5 (1904), 173-180. MR1503541

[C] Y.-G. Chen, On integers of the forms $k^{r}-2^{n}$ and $k^{r} 2^{n}+1$, J. Number Theory 98 (2003), 310-319. MR1955419 (2003m:11004) 
[CS] F. Cohen and J. L. Selfridge, Not every number is the sum or difference of two prime powers, Math. Comput. 29 (1975), 79-81. MR0376583(51:12758)

[Co] J. H. E. Cohn, Square Fibonacci numbers, etc., Fibonacci Quart. 2 (1964), 109-113. MR0161819 (28:5023)

[DG] H. Darmon and A. Granville, On the equations $z^{m}=F(x, y)$ and $A x^{p}+B y^{q}=C z^{r}$, Bull. London Math. Soc. 27 (1995), 513-544. MR1348707(96e:11042)

[E] P. Erdős, On integers of the form $2^{k}+p$ and some related problems, Summa Brasil. Math. 2 (1950), 113-123. MR0044558(13:437i)

[FFK] M. Filaseta, C. Finch and M. Kozek, On powers associated with Sierpinski numbers, Riesel numbers and Polignac's conjecture, J. Number Theory 128 (2008), 1916-1940. MR2423742

[FFKPY] M. Filaseta, K. Ford, S. Konyagin, C. Pomerance and G. Yu, Sieving by large integers and covering systems of congruences, J. Amer. Math. Soc. 20 (2007), 495-517. MR 2276778 (2007k:11016)

[GS] S. Guo and Z. W. Sun, On odd covering systems with distinct moduli, Adv. in Appl. Math. 35 (2005), 182-187. MR2152886 (2006e:11018)

[Gu] R. K. Guy, Unsolved Problems in Number Theory, Third edition, Springer, New York, 2004, Section A19, B21, F13. MR2076335 (2005h:11003)

[HS] H. Hu and Z. W. Sun, An extension of Lucas' theorem, Proc. Amer. Math. Soc. 129 (2001), 3471-3478. MR 1860478 (2002i:11019)

[IR] K. Ireland and M. Rosen, A Classical Introduction to Modern Number Theory, Second edition, Grad. Texts in Math. 84, Springer, New York, 1990. MR1070716 (92e:11001)

[LS] F. Luca and P. Stănică, Fibonacci numbers that are not sums of two prime powers, Proc. Amer. Math. Soc. 133 (2005), 1887-1890. MR2099413 (2005k:11023)

[R] P. Ribenboim, The Little Book of Bigger Primes, Second ed., Springer, New York, 2004. MR.2028675 (2004i:11003)

[S92] Z. W. Sun, Reduction of unknowns in Diophantine representations, Sci. China Ser. A 35 (1992), no. 3, 257-269. MR 1183711 (93h:11039)

[S00] Z. W. Sun, On integers not of the form $\pm p^{a} \pm q^{b}$, Proc. Amer. Math. Soc. 128 (2000), 997-1002. MR.1695111(2000i:11157)

[SY] Z. W. Sun and S. M. Yang, A note on integers of the form $2^{n}+c p$, Proc. Edinburgh Math. Soc. 45 (2002), 155-160. MR.1884609 (2002j:11117)

[W] B. M. M. de Weger, Algorithms for Diophantine Equations, CWI Tract, Vol. 65, Stichting Mathematisch Centrum, Centrum voor Wiskunde en Informatica, Amsterdam, 1989. MR 1026936 (90m:11205)

[Z] K. Zsigmondy, Zur Theorie der Potenzreste, Monatsh. Math. 3 (1892), 265-284. MR 1546236

Department of Mathematics, Zhanjiang Normal University, Zhanjiang 524048, PeoPLE'S RePUblic OF ChinA

E-mail address: kjwu328@yahoo.com.cn

Department of Mathematics, Nanjing University, Nanjing 210093, People's Republic of China and State Key Laboratory of Novel Software Technology, Nanjing UniverSity, NANjing 210093, People's Republic of China

E-mail address: zwsun@nju.edu.cn

$U R L:$ http://math.nju.edu.cn/ ${ }^{\sim}$ wsun 